\title{
Stress Distribution in the Vicinity of Slender Notches Having Arbitrary Shapes
}

\author{
Technical note covers a photoelastic investigation \\ conducted to study the stress field around the tips ot notches \\ which simulate cracks of arbitrary geometries
}

\author{
by S. A. Hussainy, H. Mohamed Hasseem and G. K. Bhagawan
}

\begin{abstract}
It was observed, by a photoelastic investigation of the stress distribution around slender notches having arbitrary configurations, that the symmetricantisymmetric and asymmetric nature of the configuration rather than the overall geometry controls the stress distribution around the notch tip. Since the peak stresses are found to be on the conservative sicle for symmetric configurations, it is suggested that the available theoretical expressions for the stress-intensity factor of an equivalent circular-arc crack be utilized for further work on crack-propagation studies.
\end{abstract}

\section{Introduction}

Many theoretical and experimental solutions are available forthe stress distribution in the vicinity of cracks with only simple geometries. 1, 2 However, solutions are not available for cracks of complex geometries which may be encountered in studies relating to crack growth. To establish the dependence of stress-intensity factor on the geometry of crack shape, a photoelastic investigation is now made to study the stress field around the tips of notches which simulate cracks of arbitrary geometries.

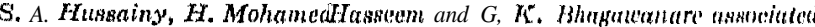
with the National Aeronautical l,aborutory, Mangalore, India.

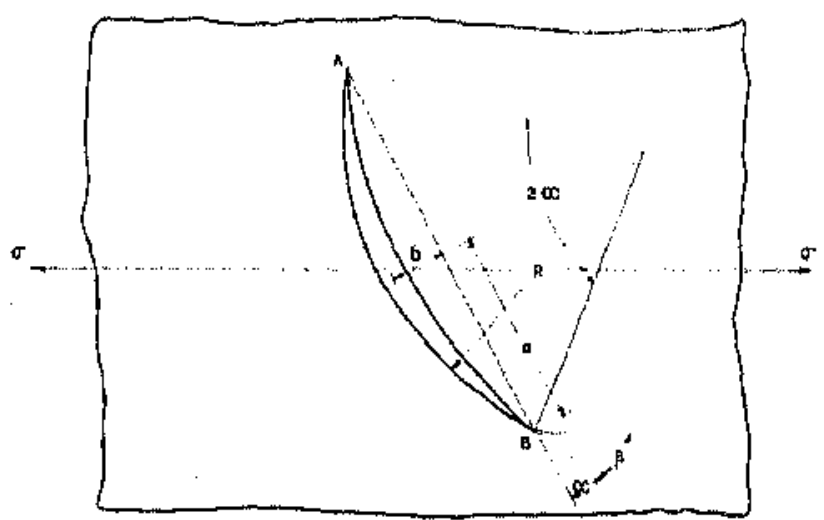

Fig. 1(a)-A circular-arc crack in an Infinite sheet subjected to uniform tension in an arbitrary direction
Before explaining the experimental work, $t$ is worthwhile re-examining the available theor ical expressions of $K$, and $K_{l}$, the stress-inte sity factors for the first and second modes, respect ely, of a circular-arc crack [Pig. 1(a)].

$K_{i}$

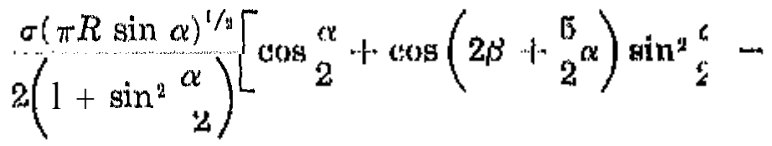

$$
\begin{aligned}
& \cos \left(2 \beta-\mathrm{I}-\frac{3 \alpha}{2}\right)\left(\operatorname{son}_{2}^{\alpha}-\sin _{2}^{\alpha} \alpha\right)-
\end{aligned}
$$

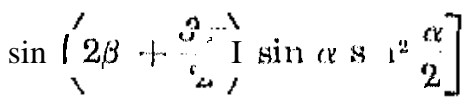

and

$K_{I I}=$

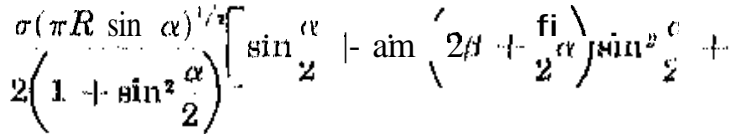

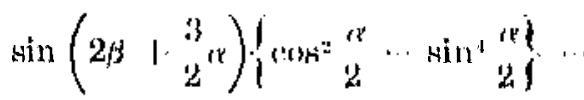

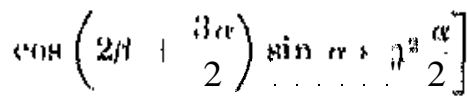

By suitulite algobrate monipulations, these an be simplified th

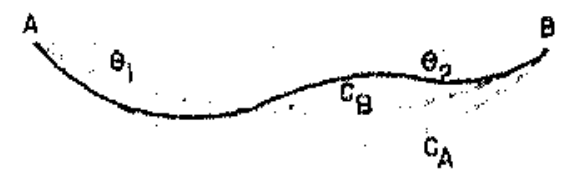

$\left(K_{\text {I }} \cdot K_{\text {III }}\right)$ AT A* $\left(K_{I}, K_{\text {II }}\right.$ ) FOR ARC $C_{A}$ WITH SLA 'E $\theta_{1}$

$\left(k_{\text {I }}, k_{\text {II }}\right)$ AT $\neq\left(k_{1}, k_{\text {II }}\right)$ FOR ARC $c_{g}$ WITH SL PE $\theta_{2}$ Flg. 1(b)-Clrcularare approximation for arbltrary er ats shapes 


$$
\begin{aligned}
& K,=f_{1}(\alpha) K_{t c h}+f_{2}(\alpha) K_{I I r h}+f_{3}(\alpha) K_{r h n} \\
& K_{H}=g_{1}(\alpha) K_{I t c h}+g_{2}(\alpha) K_{I r h}+g_{3}(\alpha) K_{r \cdot k n}
\end{aligned}
$$

where $K_{I c h}$ and $K_{I I c h}$ are the stress-intensity factors for the hypothetical chordal crack AB [Fig. 1 (a)] as defined by equation 161 of Ref. $1, K_{c h n}$ the stressintensity factor for the chordal crack with the load normal to it (i.e., $\beta=90 \mathrm{deg}$ ) and $f(\alpha), g(\alpha)$ suitable functions of the tip angle $a$.

This implies that the actual geometry away from the tips has little or no influence on the local stress condition. Based on the above observation, it is now anticipated that this may be generally true for shapes other than circular arcs. If this can be established, it would suggest a means of finding the stress-intensity factor for arbitrary crack shapes [Fig. 1(b)] to a first-order approximation.

\section{Experimental Work}

The photoelastic models were made out of $1 /$-in.thick CR 39 sheet for obtaining the isochromatics and $1 / 4$-in.-thick Perspex for obtaining isoclinics. The models were cut by using a milling machine, the speed of the cutter being $0,8 \mathrm{ft} / \mathrm{sec}$ and the central notches were made by using a jig-boring machine fitted with a specially made engraving tool to give partial-thickness cuts. The root radius of all the notch configuration (Fig. 2) was maintained at the lowest practical value of $1 / 3_{2}-i n$. , the width $1 / 10$ in., and the chordal distance between the tips $11 / 2$ in. The Perspex models were first loaded to obtain the isoclinics at different points around the notch tip. Later, all the CR 39 models were subjected to a constant tensile stress of 260 psi. The fractional fringe order at different points around the notch tip was determined by setting the circular polariscope with respect to the particular isoclinic for each point,

Tardy's compensation technique, as well as a Babinet compensator, were employed for measure-

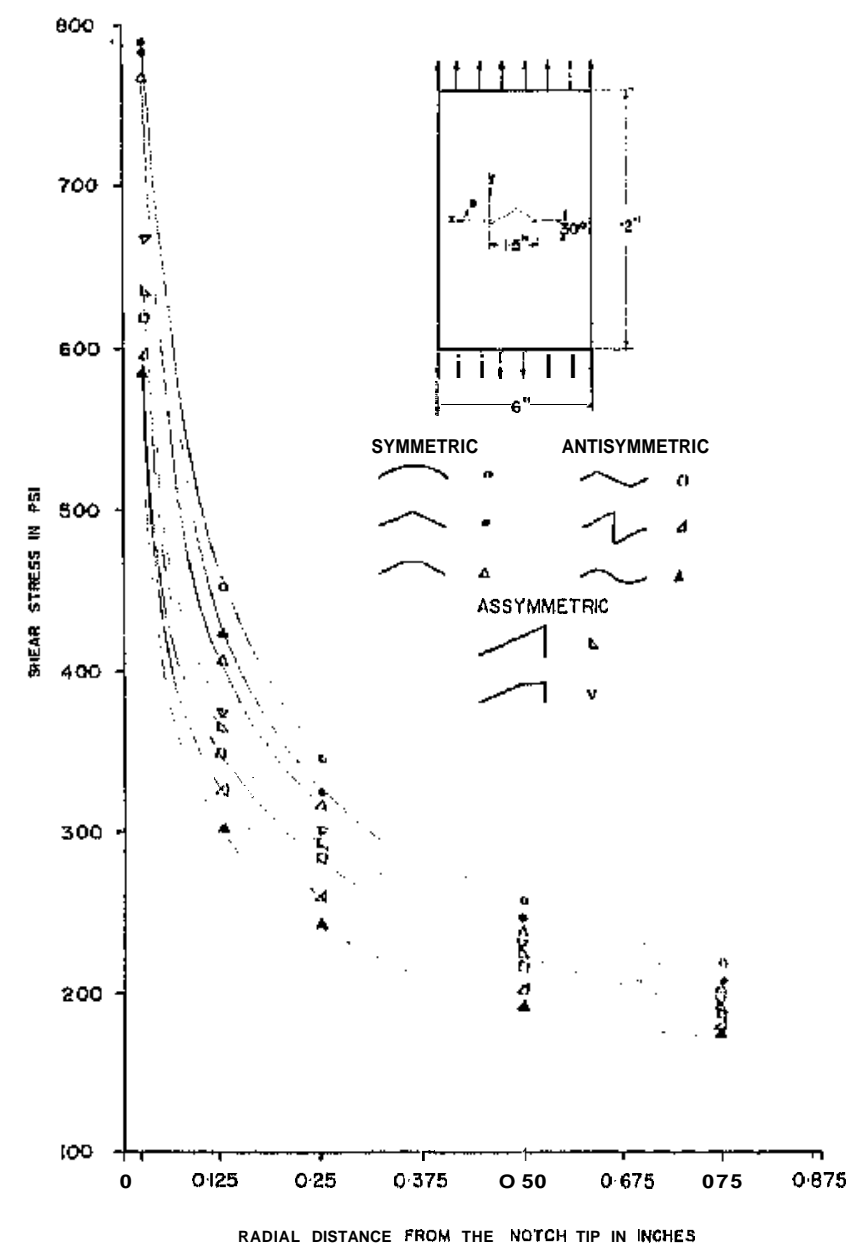

Fig. 2-Distribution of shear stress along the radial lines $(0=-90 \mathrm{deg})$

merits. The observation nearer to the notch tip was confined to distances beyond $1 / 10 \mathrm{in}$. due to time-edge effect in the models.

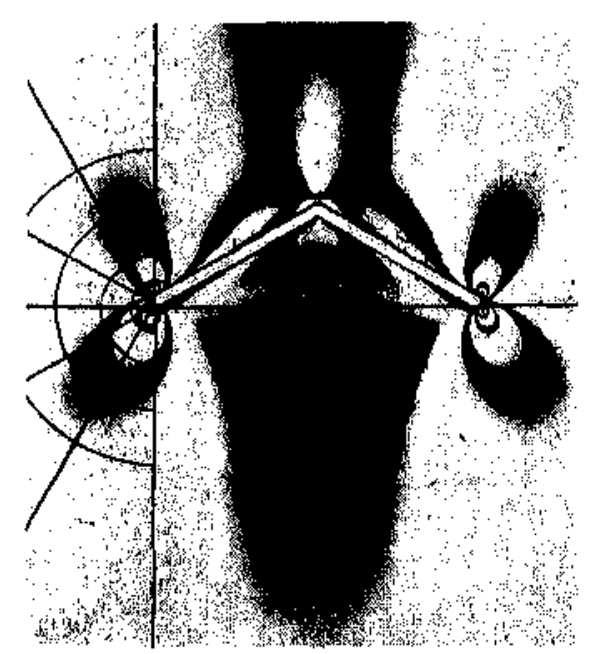

(a)

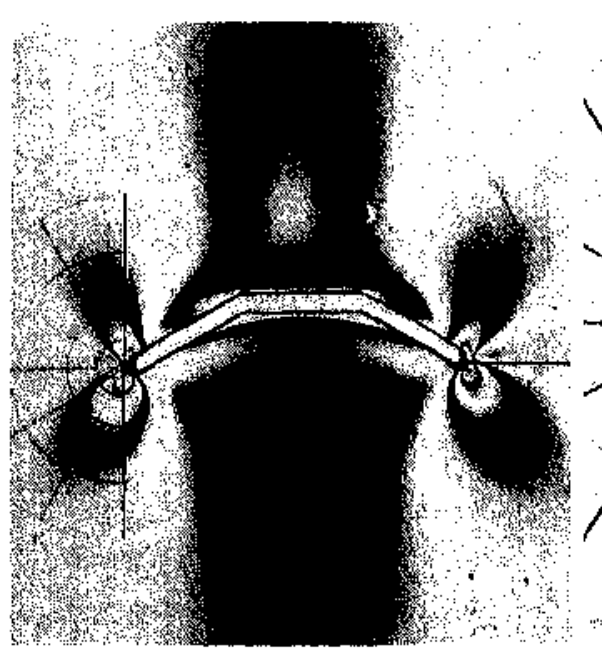

(b)

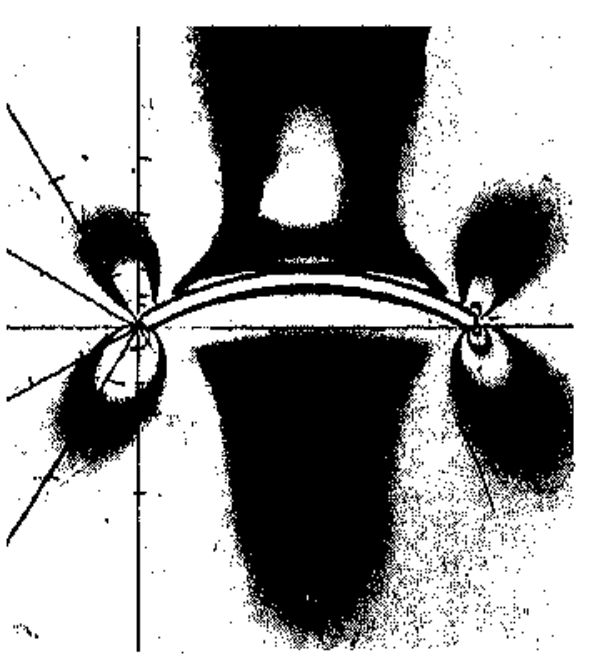

(c)

Fig. 3-Symmetric configurations (applied stress, 260 psi), Isochromatics (bright background) 


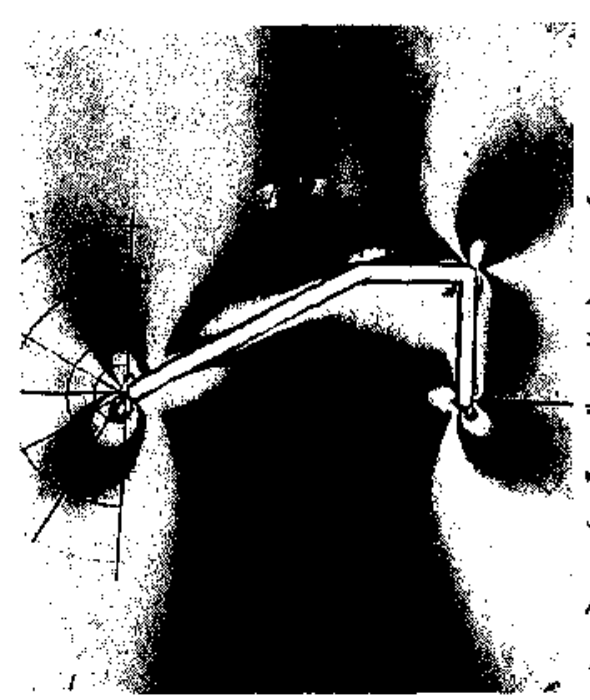

(a)

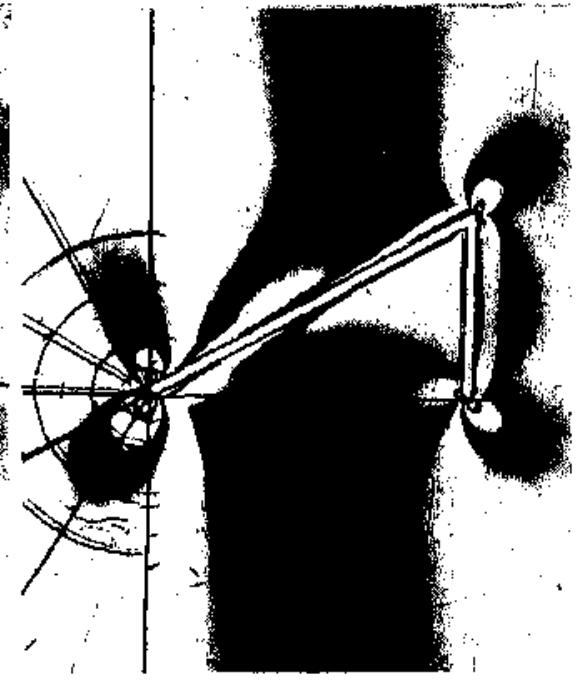

(b)
Fig. 4-Asymmetric configurations (applied stress, 260 psi). Isochromatics (bright background)

\section{Result and Discussion}

A great similarity was observed in isochromatic fringe patterns for all the configurations in the respective groups (see Figs. 3, 4 and 5). The maximum variation in the peak stress in any group is six percent.

It can be seen from the data presented that the peak stresses are relatively more for symmetric configuration rather than for the asymmetric or antisymmetric ones. At points away from the notch tip, the effect felt due to the notch is practically the same for all geometries. These results indicate that the local stress distribution is strongly dependent on the chordal distance and the general symmetric, asymmetric or antisymmetric nature of the geometry rather than the overall geometry. Thus, as a first approximation for any arbitrary crack, one can safely use the stress-intensity fuctor for an equivalent circular arc [Fig. 1(b)], the theorretical solutions of which are available in the literature. The estimates so made will always he on the conservative side.

\section{Acknowledgments}

The authors thank K. N. Rajufor suggesting this problem, N. Narayana Iyer for making the models, and A. Subramaniam for his help in conducting the experiments.

\section{References}

1. I'aris, P. (',., and Sih, G. G., "Stress Analysis of Crathis." As'I'MI STP. 381, 31.0.' (1964)

2. Wells, A, A., and POAt, /J., "The Dymamic sirese Dintribution fiur rounding a Ruming Crack on Holu in a Flat Plate." Proc. SHSA, XVI (L), 619$) 2(10,5)$.

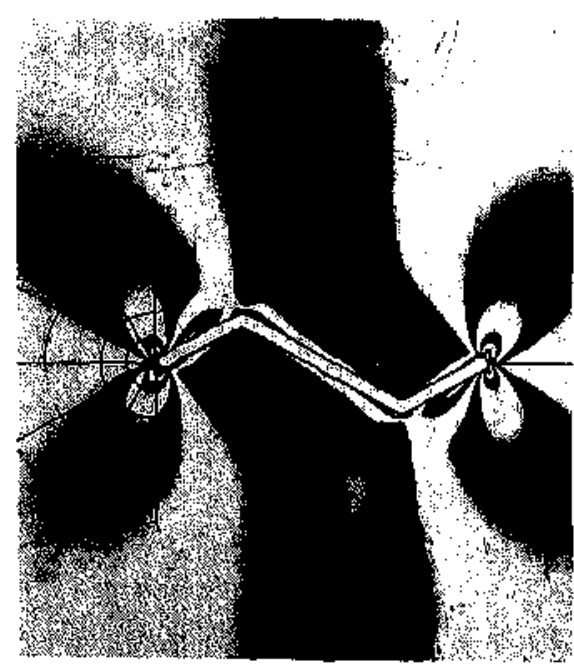

(a)

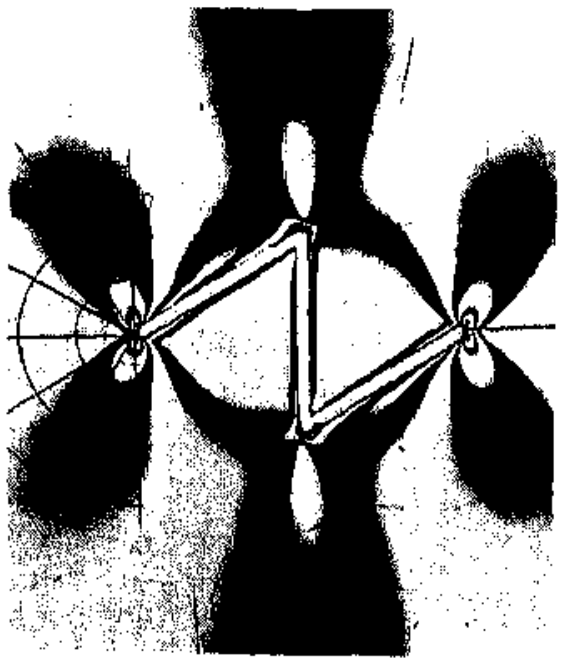

(b)

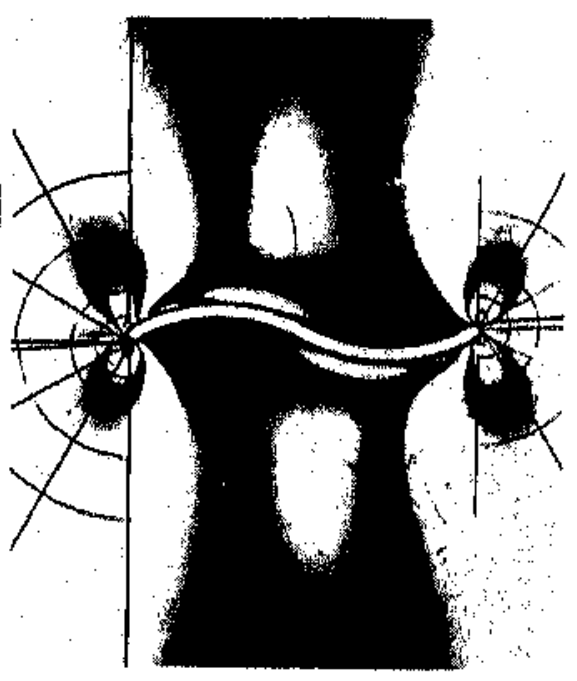

(c)

Fig. 5-Antisym metric configurations (applied stress, 260 psi). Isochromatics (bright background) 